\title{
Simple One-Pot Synthesis of Fully Biobased Unsaturated Polyester Resins Based on Itaconic Acid
}

Vesna V. Panic, ${ }^{\dagger}$ Sanja I. Seslija, ${ }^{\ddagger}$ Ivanka G. Popovic, ${ }^{\S}$ Vuk D. Spasojevic,, Aleksandar R. Popovic, ${ }^{\perp}$ Vladimir B. Nikolic, ${ }^{\prime}$ and Pavle M. Spasojevic*, $*, \dagger \odot$

${ }^{\dagger}$ Innovation Center of Faculty of Technology and Metallurgy, University of Belgrade, 4 Karnegijeva Street, 11000 Belgrade, Serbia

${ }^{\ddagger}$ Institute of Chemistry, Technology and Metallurgy, University of Belgrade, 12 Njegoseva Street, 11000 Belgrade, Serbia

${ }^{\S}$ Faculty of Technology and Metallurgy, University of Belgrade, 4 Karnegijeva Street, 11000 Belgrade, Serbia

"Vinca Institute of Nuclear Sciences, University of Belgrade, P.O. Box 522, 11001 Belgrade, Serbia

${ }^{\perp}$ Faculty of Chemistry, University of Belgrade, Studentski trg 12-16, 11000 Belgrade, Serbia

\# Innovation Center, Faculty of Chemistry, University of Belgrade, Studentski trg 12-16, 11000 Belgrade, Serbia

${ }^{\nabla}$ Faculty of Technical Sciences, University of Kragujevac, 65 Svetog Save Street, 32000 Cacak, Serbia

Supporting Information

ABSTRACT: For the preparation of fully biobased unsaturated polyester resins (UPRs), the replacement of styrene with alternate nonpetroleum-based monomers turned out to be one of the most challenging tasks. Its complexity lies in the fact that reactive diluents $(\mathrm{RD})$ have to have low viscosity and volatility, good compatibility with prepolymer, and capability to homopolymerize and copolymerize with its unsaturations. In this context, we directed our efforts to develop fully biobased UPRs using the dialkyl itaconates as an alternative to styrene. Therefore, a series of $100 \%$ biobased UPRs were prepared from itaconic acid and 1,2-propandiol and diluted by dialkyl itaconates. The resins were characterized by Fourier transform

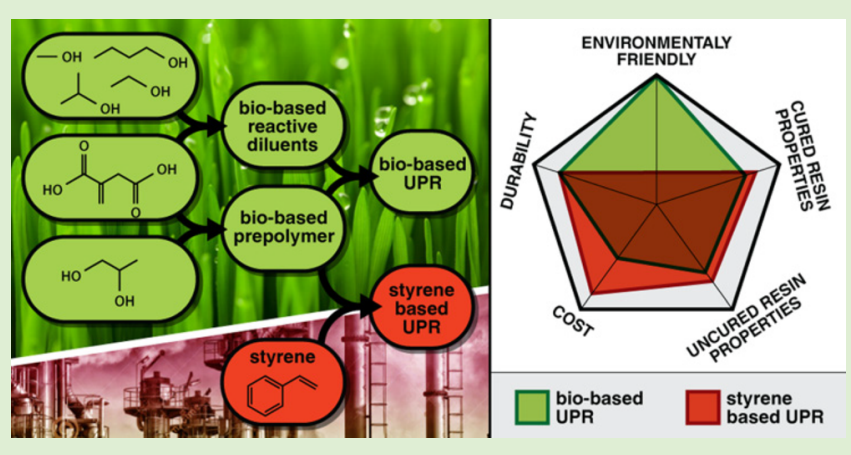
infrared spectroscopy, NMR, volatility, and viscosity measurements, while the cured samples were characterized by dynamic mechanical properties, thermomechanical analysis, thermogravimetric analysis data, and tensile tests. The influence of RD structure on the properties of cured samples was discussed in detail. It was shown that the prepared resins had evaporation rates of dialkyl itaconates of several orders of magnitude less compared to styrene. The cured resins with dimethyl itaconate showed comparable or even better thermal and mechanical properties compared to the one with styrene. This investigation showed that itaconic acid and dialkyl itaconates are promising bioresources for the preparation of fully biobased UPRs for mass consumption.

\section{INTRODUCTION}

Fundamental shift to the biobased production and economy represents an everyday challenge. We are thereby witnessing a different hurdle and level of success in different areas. One of the promising areas involves the chemically manufactured products for massive consumption, such as unsaturated polyester resins (UPRs).

Nowadays, the main issue related to their conventional, petrochemical-based formulations comes from the use of styrene as reactive diluent. Because of its high volatility it has been recognized as a hazardous air pollutant by the Environmental Protection Agency. ${ }^{1}$ Also, its carcinogenic nature has been foreseen by the United States Department of Health and Human Services through the National Toxicology Program. ${ }^{2}$ Besides the health issue, the search for a styrene replacement has become industrially and fundamentally attractive due to increase in concern about the greenhouse effect and the unpredictable oscillation of the crude oil prices. ${ }^{3}$
Therefore, a lot of effort has been invested in finding a styrene replacement among the reactive diluents $(\mathrm{RD})$ derived from renewable and/or sustainable resources. ${ }^{4,5}$

Trying to meet requirements related to the final properties of the desired material, it has been considered that $\mathrm{RD}$ should keep the favorable properties of styrene. Many reported attempts to find an appropriate biobased reactive diluent as a styrene replacement testify to the complexity of the problem.

Fatty acid-based monomers are among the most widely investigated biobased $\mathrm{RD}$. Although they are almost nonvolatile, the moderately high viscosity and low glass transition temperature of the cured resins have restricted their development. $^{6-8}$ Much effort has also been put in the potential use of aromatic biobased monomers, which are mainly derived from

Received: June 15, 2017

Revised: October 23, 2017

Published: October 24, 2017 
lignin. $^{9-11}$ However, their viscosity, which is $25-40$ times larger compared to styrene, limits their use as a styrene replacement due to the processing issues. Cousinet et. al have investigated vinyl levulinate (VL) as a potential replacement for styrene. ${ }^{12}$ Although some promising results have been reported, VL-based UPRs have exhibited lower $\alpha$-relaxation temperature, elastic moduli at the rubbery plateau, and mechanical properties determined by the three points bending tests. Research has been done regarding the use of unsaturated cyclocarbonates as reactive diluents, too. ${ }^{13}$ The unsaturated cyclocarbonate monomers have been proven as less harmful compounds but their lower reactivity has made them unsuitable as reactive diluents for UPRs. The most promising results have been obtained for biobased methacrylates., ${ }^{3,14}$ Biobased butanedioldimethacrylate UPR has shown comparable properties to styrene-cured UPR but with poor ductility and resilience. This has required new efforts in finding an additional codiluent which would exhibit a good miscibility with butanedioldimethacrylate and increase resin's ductility and resilience at same time.

Despite considerable work and numerous attempts to overcome the described issues, a suitable biobased replacement for styrene has not been identified yet. The peculiar reactivity of the prepolymer fumarate group's double bonds on $\mathrm{RD}$ may be the one of the main reasons for this state of affairs. Basically, fumarate groups copolymerize well with styrene, ${ }^{15}$ but copolymerization between fumarate groups and many biobased RDs has turned to be problematic. ${ }^{16,17}$ Then, could the new approach involving the replacement of styrene but also the prepolymer with compatible biobased compounds be the solution?

The aim of this research is directed toward the synthesis and characterization of fully biobased unsaturated polyester resins, competitive in price and performance and adaptable to the existing manufacturing processes. The proposed approach involves the replacement of styrene using the esters of itaconic acid as reactive diluents, which are far less toxic and volatile compared to styrene and therefore present lower threat to human health and environment. In order to increase compatibility between the prepolymer and $\mathrm{RD}$, the prepolymer is synthesized using itaconic acid (IA) and 1,2-propylene glycol.

The molecule of choice, itaconic acid, is a diacid containing an active 1,1-disubstituted vinyl group. IA is not a newfound chemical but it is experiencing a kind of renaissance; after 50 years, new approaches enabled increasing exploitation of its properties. that is, unsaturated nature coupled with two carboxylic groups.

IA and its derivatives are under extensive consideration in developing biobased UPR because of unsaturation but also favored fermentative production from carbohydrates. ${ }^{18}$ Unlike similar biobased acids it possess reactivity that can be controlled, and recently even genetic modification has been applied on some plants to produce more IA. It is no surprise then that it is recognized as one of the top 12 biobased molecules by the U.S. Department of Energy. Because of its resemblance to maleic/fumaric acid it is a highly interesting chemical building block for UPR prepolymer. ${ }^{19,20}$ Although, the current market of products derived from itaconic acid is still rather small and only a few applications are yet established due to the high production costs, it is expected to find an appropriate production pathway very soon. ${ }^{21}$

\section{EXPERIMENTAL SECTION}

Materials. The itaconic acid, 1,2-propandiol, hydroquinone, and the styrene monomer were supplied from Sigma-Aldrich. The dialkyl itaconates used in the synthesis of UPRs were obtained via the esterification reaction of itaconic acid and corresponding alcohol (methanol, ethanol, $n$-butanol, and isopropyl alcohol), as described earlier. $^{23,24}$ All of the reactants employed in the synthesis of dialkyl itaconates were supplied by Merck. For the preparation of cured resins, methyl ethyl ketone peroxide (MEKPO) (Sigma-Aldrich) was used as an initiator. All chemicals were used as received.

Synthesis. The prepolymer was synthesized by the melt polycondensation of itaconic acid with 1,2-propandiol (the mole ratio of itaconic acid toward 1,2-propandiol was 1:1.05). The components were mixed in a $500 \mathrm{~mL}$ four-necked round-bottom flask connected with a stirrer, a thermometer, a Dean-Stark, and a $\mathrm{N}_{2}$ gas inlet. Hydroquinone, $100 \mathrm{ppm}$, was added into the reaction mixture at the beginning of the reaction as free radical scavenger, while titanium butoxide $\left(\mathrm{Ti}(\mathrm{OBu})_{4}\right)(1.5 \mathrm{wt} \%)$ was used as the catalyst. The reaction was carried out within a temperature range of $110-190{ }^{\circ} \mathrm{C}$ under a nitrogen atmosphere wherein the temperature was raised for $10{ }^{\circ} \mathrm{C}$ per hour. The reaction was carried out until the acid value reached 50. The resin was allowed to cool to $110^{\circ} \mathrm{C}$ under a nitrogen atmosphere; then, it was dissolved in reactive diluents, corresponding dialkyl itaconate or styrene $(40 \% \mathrm{w} / \mathrm{w}$ with respect to the resin). The UPR resin with styrene as reactive diluent was synthesized for comparison.

This way synthesized UPRs and MEKPO $(2.5 \% \mathrm{w} / \mathrm{w})$ were mixed together and mixed up until the mixture was homogeneous. Afterward, the mixtures were poured into Teflon molds, placed in an air oven, and then left at $80^{\circ} \mathrm{C}$ for $3 \mathrm{~h}$ and at $120^{\circ} \mathrm{C}$ for an additional hour to cure.

The acid value (AV) was defined as the number of milligrams of $\mathrm{KOH}$ needed to neutralize $1 \mathrm{~g}$ of resin and was measured according to ASTM D465-01. Around $0.5 \mathrm{~g}$ of resins was titrated with a $\mathrm{KOH}$ solution in ethanol $(0.1 \mathrm{~mol} / \mathrm{L})$.

\section{METHODS}

Prepolymer Characterization. NMR spectroscopy was used for addressing the chemical structure of the prepolymer. The experiment was performed on a Bruker DMX-400 spectrometer equipped with a 5 $\mathrm{mm}$, inverse detection, z-gradient probe. The ${ }^{1} \mathrm{H}$ and ${ }^{13} \mathrm{C} N M R$ spectra (at 400 and $100 \mathrm{MHz}$, respectively) were measured at room temperature using $\mathrm{CDCl}_{3}$ as the solvent.

The transmission IR spectra of the prepolymer were obtained on a Bomem MB100 FTIR spectrophotometer by collecting and averaging 30 scans at a resolution of $4 \mathrm{~cm}^{-1}$. A liquid film of the sample was pasted between two $\mathrm{ZnS}$ plates mounted on a specific sample holder located in the FTIR instrument. ZnS plates were used because their absorbance is low between 800 and $4000 \mathrm{~cm}^{-1}$.

The molar mass of the prepolymer was determined by gel permeation chromatography, GPC, at $30{ }^{\circ} \mathrm{C}$ using a Waters 1500 Series instrument fitted with four analytical columns (Waters HR 2, HR 3, HR 4, and HR 5E) and an RI detector. Chloroform was used as the mobile phase at a flow rate of $1 \mathrm{~mL} \mathrm{~min}{ }^{-1}$. Waters Breeze software was used for data acquisition and linear poly(styrene) standards were used for calibration.

Viscosities were measured isothermally at $25{ }^{\circ} \mathrm{C}$ using Discovery HR-2 (TA Instruments, New Castle, DE) in Peltier plate $\left( \pm 0.1{ }^{\circ} \mathrm{C}\right.$ error) geometry. The sample (around $0.05 \mathrm{~mL}$ ) was loaded in a 20 $\mathrm{mm} 1^{\circ}$ steel cone with a truncation gap of $25 \mu \mathrm{m}$. The shear rate was increased stepwise from 1 to $100 \mathrm{~s}^{-1}$, collecting 21 data points to observe any non-Newtonian behavior. At the given shear rate, the shear stress was measured every $2 \mathrm{~s}$. The data were recorded when the shear rate was stabilized with up to $5 \%$ tolerance for three consecutive points. UPRs were measured in triplicate and the viscosities were averaged and reported.

UPRs Characterization. Evaporation of RD was investigated by measuring the weight loss with a TGA701 thermogravimetric analyzer. Around $200 \mathrm{mg}$ of the UPR sample was placed in an aluminum pan 
Scheme 1. Titanium(IV) Butoxide Catalyzed Polycondensation of Itaconic Acid and 1,2 Propandiol with Hydroquinone ${ }^{a}$

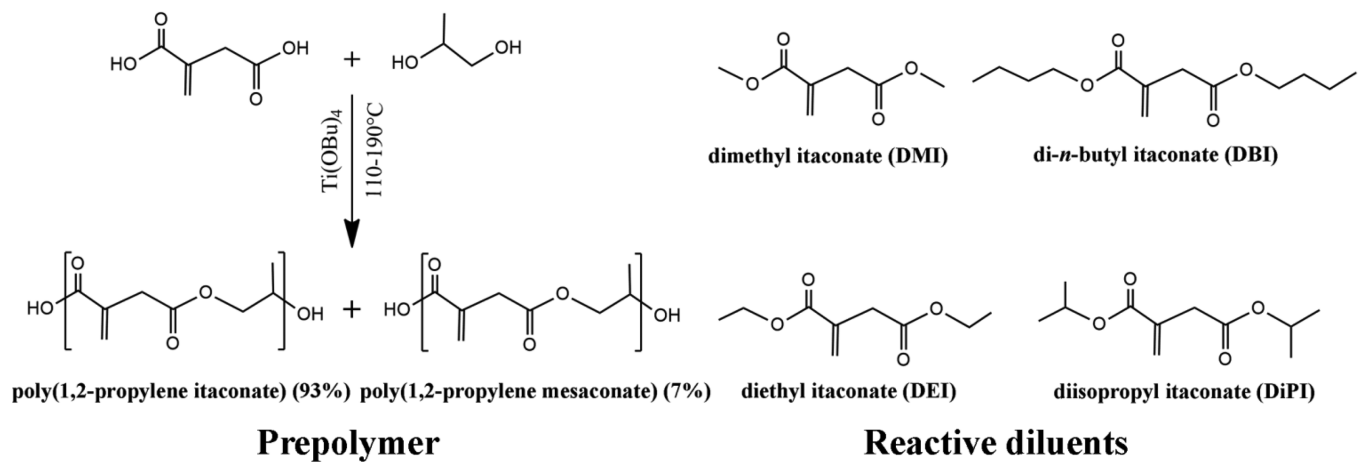

${ }^{a}$ (Left) as the radical inhibitor and (right) as bio-derived reactive diluents.

and left at $30{ }^{\circ} \mathrm{C}$ under nitrogen $\left(10 \mathrm{~mL} \mathrm{~min}{ }^{-1}\right.$ gas flow rate). The weight loss was monitored within $15 \mathrm{~h}$.

The dynamic mechanical properties (DMA) of the UPR samples were analyzed by a Discovery HR-2 (TA Instruments, New Castle, $\mathrm{DE})$. The samples were exposed to fixed strain amplitude of $0.1 \%$ and angular frequency of $1 \mathrm{~Hz}$ in the temperature range from 25 to $200^{\circ} \mathrm{C}$. The measured data were storage modulus $\left(G^{\prime} / \mathrm{GPa}\right)$, loss modulus $\left(G^{\prime \prime} / \mathrm{MPa}\right)$, and damping factor $(\tan \delta)$, while glass transition temperature $\left(T_{\mathrm{g}}\right)$ was determined as the temperature where $\tan \delta$ had a maximum value. All results are obtained from second heating cycle. The thermo-mechanical analysis (TMA) was conducted in an expansion mode using Q400 TA Instrument, U.K. The samples were subjected to receive a constant load of $10 \mathrm{mN}$ and expansion measurements were carried out from 0 to $100{ }^{\circ} \mathrm{C}$ with the heating rate of $10{ }^{\circ} \mathrm{C} \mathrm{min}{ }^{-1}$ under the argon atmosphere $\left(100 \mathrm{~mL} \mathrm{~min}^{-1}\right)$. The cured samples surface properties were investigated on the same instrument by means of the compression mode. The tests were done at ambient temperature with constant speed of $2.5 \mu \mathrm{m} \mathrm{min}{ }^{-1}$. Maximal applied stress was very low $(0.075 \mathrm{MPa})$, so only the sample surface was affected.

The tensile properties were analyzed using an universal testing machine, Instron 3300 (Instron, U.S.A.). The specimens were fixed by mechanical clamps and the measuring speed was $0.5 \mathrm{~mm} \mathrm{~min}^{-1}$. For each sample, five measurements were done. The average values of the break stress and break stroke strain, as well as the standard deviations, were determined. The elastic moduli were determined from the linear part of the stress/strain curve.

Thermogravimetric analyses were performed under dynamic air in the temperature range $25-1000{ }^{\circ} \mathrm{C}$ using a TGA701 thermogravimetric analyzer. The air flow rate was $10 \mathrm{~mL} \mathrm{~min}^{-1}$, while the heating rate was $10^{\circ} \mathrm{C} \mathrm{min}^{-1}$. The weight of the samples was approximately 25 mg.

The density of cured resins was measured using glass picnometer (Gay-Lussac type). The samples were ground and sieved to $1 \mathrm{~mm}$ size and placed in the picnometer bottle filled with water.

\section{RESULTS AND DISCUSSION}

Prepolymer Characterization. Dialkyl itaconates of a different structure (dimethyl itaconate (DMI), diethyl itaconate (DEI), diisopropyl itaconate (DiPI), and dibuthyl itaconate (DBI)) were used as reactive diluents instead of styrene in UPRs formulations. To obtain fully biobased UPRs and increase the compatibility between $\mathrm{RD}$ and prepolymer, the latter was prepared via polyesterification of itaconic acid and propylene glycol (Scheme 1).

As can be seen by the ${ }^{1} \mathrm{H}$ NMR spectra, Figure 1, the prepolymer synthesis was successful with the signals at 5.67 and $6.27 \mathrm{ppm}$ indicating the survival of the double bonds. The presence of signals at 2.20 and $6.61 \mathrm{ppm}$ indicated that around $7 \%$ of the itaconate moieties had isomerized to the mesaconate

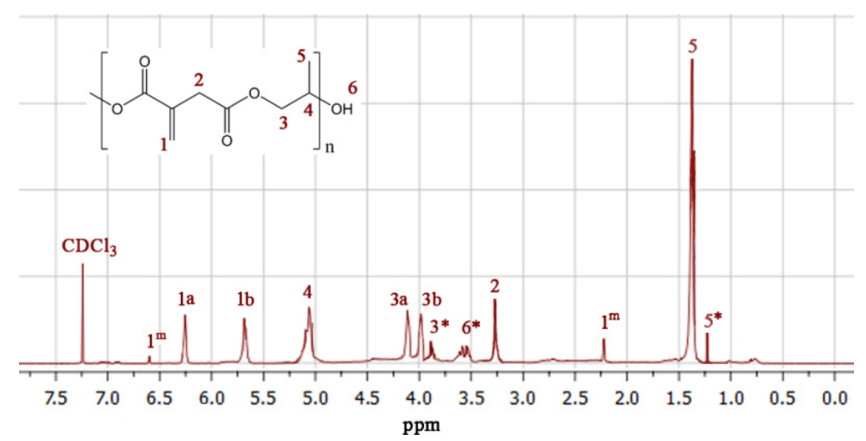

Figure 1. ${ }^{1} \mathrm{H}$ NMR spectra of prepolymer. Peaks marked with * are the protons of the end-groups; peaks marked with ${ }^{\mathrm{m}}$ are the protons of the mesaconate moiety; $1 \mathrm{a}$ and $1 \mathrm{~b}$ are for each one of the germinal protons on the itaconate double bond and are nonequivalent to one another.

equivalent. ${ }^{22}$ The ${ }^{1} \mathrm{H}$ NMR spectroscopy end-group analysis suggested molecular weight of $1324 \mathrm{~g} \mathrm{~mol}^{-1}$. However, it must be noted that signal resolution was poor as a result of broadening. The molar mass determined by GPC analysis agreed well with the molar mass predicted by ${ }^{1} \mathrm{H}$ NMR. The $\bar{M}_{\mathrm{n}}$ was found to be $1254 \mathrm{~g} \mathrm{~mol}^{-1}$ while weight-average molar mass $\left(\overline{M_{\mathrm{w}}}\right)$ was $3234 \mathrm{~g} \mathrm{~mol}^{-1}$. These values were expected and are in accordance with literature data. ${ }^{18,22}$

Although, Tang et al. claimed that addition of even low amounts of hydroquinone omitted gelation of $\mathrm{IA}^{4}{ }^{4}$ in this research we found out that gelation still occurs at lower acid value ( $\mathrm{AV}$ below 50) when performing polycondensation at elevated temperatures $\left(180-220{ }^{\circ} \mathrm{C}\right)$. The susceptibility to chain branching and eventual cross-linking during unsaturated polyester synthesis when itaconic acid is used as substrate has been claimed by many authors. ${ }^{4,18,21-23}$ Even if branching occurs at low level, without further cross-linking and gelation, the properties of obtained prepolymer are usually inadequate to be used for UPR preparation. Namely, the viscosity of so prepared UPR is generally very high and thus unsuitable for many application methods. Furthermore, curing of this resin would result in formation of inhomogeneous network with poor mechanical properties. Therefore, a detail investigation of prepolymer structure is needed to determine if any chain branching occurred. Literature suggests the mode of branching occurs via the Ordelt reaction, an acid-catalyzed $\mathrm{C}=\mathrm{C}$ bond saturation by an alcohol. ${ }^{4,18,21-23}$ According to the mechanism of the Ordelt reaction, proposed by Farmer et al., ${ }^{22}$ hydroxyl adds solely at the $\beta$-position of the unsaturated ester (Scheme 
2). The appearance of the broad region from $2.5-3.0 \mathrm{ppm}$ attributed to the $\mathrm{CH}$ and $\mathrm{CH}_{2}(\mathrm{~A}$ and $\mathrm{B}$ ) on the itaconate

Scheme 2. Ordelt Reaction, $\mathrm{C}=\mathrm{C}$ Bond Saturation by a Diol via $\beta$-Addition<smiles></smiles>

moiety of the polymer backbone indicate $\beta$-addition. Absence of signals in the region from 2.5 to $3.0 \mathrm{ppm}$ in Figure 1 indicated that branching did not occur during prepolymer synthesis. Also, new peaks, attributed to the $\mathrm{CH}_{2}$ and $\mathrm{CH}$ on the former diol section following ether formation, were not observed.

UPRs Characterization. The properties of uncured UPRs, such as their viscosity and $\mathrm{RD}$ evaporation are of a great importance because they govern and limit their usage potential. For instance, the viscosity of the uncured resins determines their mode of application such as open molding, vacuum infusion, injection, brushing, spraying, and so forth. One of the main issues regarding the synthesis of fully biobased UPRs is in particular their very high viscosity. ${ }^{3}$ Thus, understanding the relationship between the resin structure and its viscosity is essential for adjusting the properties of the resin to gain optimal performances. The viscosity curves of synthesized resins are shown in Figure 2.

The viscosity of the obtained resins varied from around 4800 $\mathrm{mPas}$ for DMI to $850 \mathrm{mPas}$ when S, DiPI, and DBI were used as $\mathrm{RD}$. Higher viscosity of the resin with DMI was expected due to the stronger polar interactions between the carbonyl groups of DMI and prepolymer. These interactions were hindered with an increase in the alkyl side chain of RD. Also, longer alkyl side chain omitted the aligning of macromolecular chains which further decreased the viscosity values. The viscosity of commercial UPRs varies from around $1000 \mathrm{mPas}$ for spraying to $10000 \mathrm{mPas}$ for gel coats. The obtained viscosity results were acceptable and indicated that investigated itaconate-based resins could be commercialized.

The evaporation of reactive diluents is one of the main environmental and health issues of UPRs. The rate of $R D$ evaporation depends on many factors, such as the nature of $\mathrm{RD}$, the type of resin, application process, application equipment used and tool design and configuration. In order to anticipate the volatility of UPRs the evaporation rate of the synthesized UPRs was examined and compared with resin diluted with styrene. Taking into consideration possible interactions between prepolymer and $\mathrm{RD}$ we decided to investigate the evaporation of $\mathrm{RD}$ from UPR instead of commonly applied monitoring of volatility of pure $\mathrm{RD}$. It was assumed that this approach would give more realistic simulation of the $\mathrm{RD}$ evaporation in real conditions. The evaporation curves are shown in Figure 2. As can be seen, the styrene evaporation was much higher compared to the itaconates. More than $82 \%$ of styrene evaporated after $15 \mathrm{~h}$ at $30^{\circ} \mathrm{C}$, while the evaporation of the itaconates was between 5 and $9.5 \%$, for DMI and DiPI, respectively. These results indicated that itaconates could be used as low volatile RD.

Cured UPRs Characterization. Bearing in mind that UPRs are widely used and highly exposed to various physical, chemical, and, in some cases, extreme environmental conditions, it is very important to perform in detail the analysis of the physical and chemical properties of the cured samples.

The gel content is one of the critical indicators for evaluating the performance of the cured UPRs. As seen in Table 1, all of the cured UPRs presented high gel content ranging from 93.7 to $98.6 \mathrm{wt} \%$. The cured UPRs showed decreased gel content with the increase in the ester alkyl group of $\mathrm{RD}$. These results were expected due to the decrease in the $\mathrm{RD}$ reactivity with the increase in ester alkyl group. Namely, due to the steric hindrance the reactivity of the reactive diluent molecules toward the prepolymer is lower compared to the reactivity toward the other reactive diluent molecules. Sato et al. have investigated the polymerization reactivity of dialkyl itaconates consisting of primary alkyl esters and found that their reactivity decreased gradually with the increasing carbon number of the ester alkyl group..$^{24,25}$

The dynamic mechanical analysis was used to investigate the thermomechanical properties of the cured resins in order to establish the effects of the constituent's structure on the properties of the thermosets. The dependencies of the storage modulus $\left(G^{\prime}\right)$ and loss tangent $(\tan \delta)$ of the cured resins on temperature are presented in the Supporting Information (Figure S4). The $G^{\prime}$ characterizes the stiffness of viscoelastic material and it is proportional to the energy stored under cyclic load, while $\tan \delta$ is a measure of how well material absorbs and dissipates energy. The commercial resins used in the
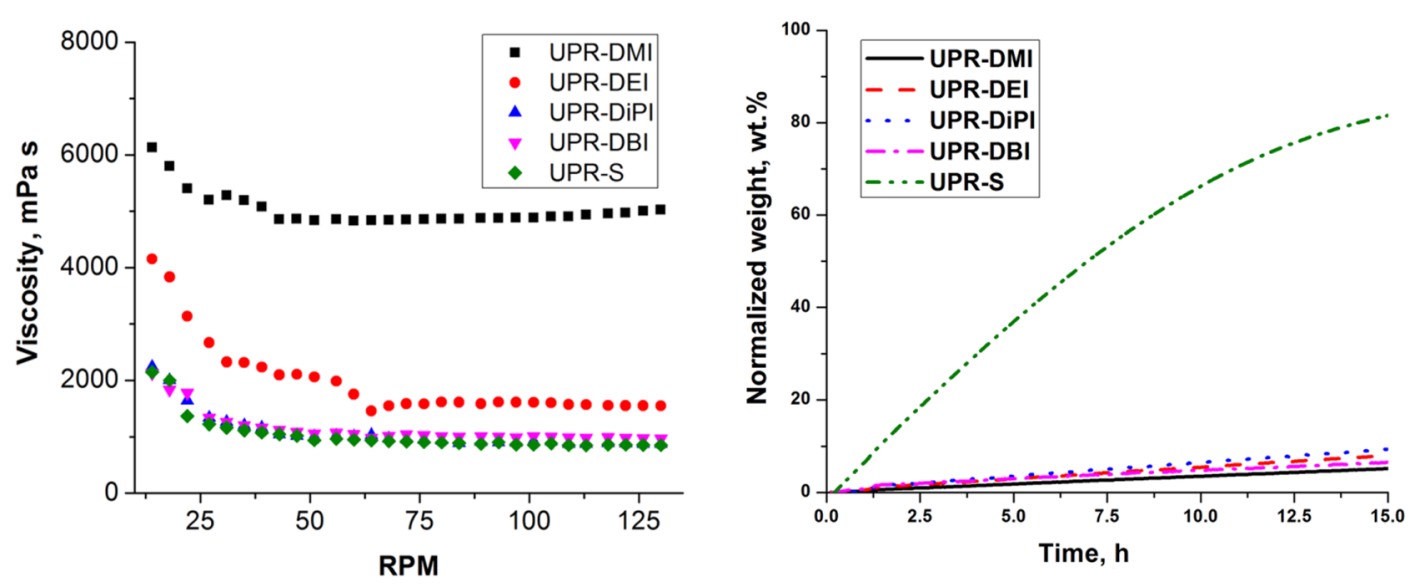

Figure 2. Viscosity of synthesized UPRs (left) and evaporation of reactive diluent (right). 
Table 1. UPRs Storage Moduli ${ }^{a}$

\begin{tabular}{|c|c|c|c|c|c|c|c|c|}
\hline sample & $G^{\prime}, \mathrm{GPa}$ & $G_{185^{\circ} \mathrm{C}}^{\prime} \mathrm{MPa}$ & $n_{\mathrm{e}}, \mathrm{mol} \mathrm{m}^{-3}$ & $\nu$ & $T_{\mathrm{g}},{ }^{\circ} \mathrm{C}$ & $\rho_{25}^{\circ} \mathrm{C}, \mathrm{g} \mathrm{dm}^{-3}$ & $\rho_{175^{\circ} \mathrm{C}}, \mathrm{g} \mathrm{dm}^{-3}$ & gel content, \% \\
\hline UPR-S & 1.23 & 30.1 & 2695 & 4.6 & 153 & 1.340 & 1.281 & 98.6 \\
\hline UPR-DMI & 1.41 & 22.1 & 1974 & 4.3 & 118 & 1.376 & 1.328 & 98.3 \\
\hline UPR-DEI & 1.06 & 15.8 & 1413 & 4.8 & 104 & 1.322 & 1.268 & 97.3 \\
\hline UPR-DiPI & 0.776 & 11.6 & 1040 & 5.4 & 96 & 1.252 & 1.202 & 94.5 \\
\hline UPR-DBI & 0.368 & 6.0 & 535 & 9.1 & 65 & 1.262 & 1.179 & 93.7 \\
\hline
\end{tabular}

${ }^{a}$ At $25^{\circ} \mathrm{C}\left(G^{\prime}\right)$ and $185^{\circ} \mathrm{C}\left(G^{\prime} 185^{\circ} \mathrm{C}\right)$, crosslink density $\left(n_{\mathrm{e}}\right)$, the average number of structural units between crosslinks $(\nu), T_{\mathrm{g}}$, densities at $25^{\circ} \mathrm{C}$ $\left(\rho_{25^{\circ} \mathrm{C}}\right)$ and calculated densities at $175^{\circ} \mathrm{C}\left(\rho_{175^{\circ} \mathrm{C}}\right)$.

\section{Chart 1. Potential Structure of the Cured UPRs ${ }^{a}$}

a)

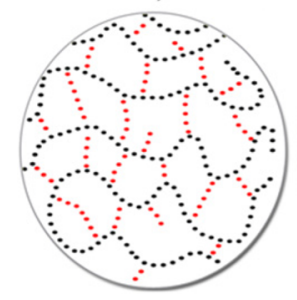

b)

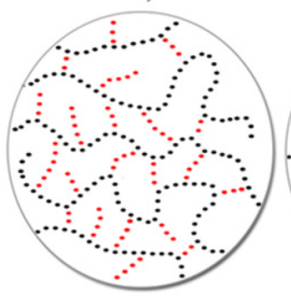

c)

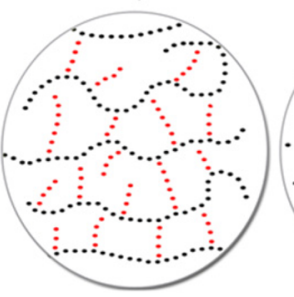

d)

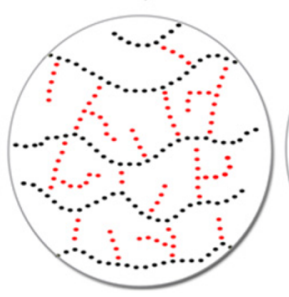

e)

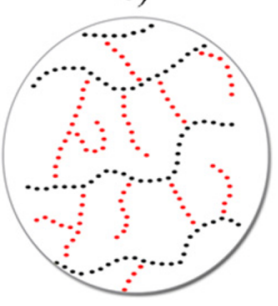

a (a) UPR-S, (b) UPR-DMI, (c) UPR-DEI, (d) UPR-DiPI, and (e) UPR-DBI; black dots represent prepolymer and red dots represent reactive diluent.

production of high-performance composite materials usually have $T_{g}$ in the range of $100-150{ }^{\circ} \mathrm{C}$ and $G^{\prime}$ in the range of $2-3$ GPa. ${ }^{14}$ The values of $G^{\prime}$ at room temperature and $T_{\mathrm{g}}$ determined as a peak of $\tan \delta$ are presented in Table 1 .

The $G^{\prime}$ in the glass region is primarily dictated by the strength of intermolecular forces existing between polymer chains and the arrangement of the polymer chains packing. ${ }^{26}$ The highest value of $G^{\prime}$ was obtained for UPR-DMI, while with the increase in the alkyl chain length of the itaconate side groups the values of $G^{\prime}$ decreased. The decrease could be explained by the poorer chain packing coming from the bulky side groups. Also, larger side groups sterically hindered the electrostatic interaction of carbonyl groups which decreased the overall intermolecular interactions. On the other hand, very small methyl group of DMI slightly affected the chain packing while at the same time, as electron donor group, it increased the electrostatic interactions of carbonyl group.

A more detailed comparison of the storage moduli at glassy and rubbery state showed a lower decrease of storage modulus of UPR-S compared to the other resins. The storage modulus of UPR-S decreased 40 times compared to around 65 times for other resins. This could be explained by the interaction of dangling chains. Namely, polystyrene chains were more rigid compared to poly(alkyl itaconate) chains due to high rigidity of phenyl ring. Hence, poly(alkyl itaconate) chains were more susceptible to physical entanglement and self-assembling of nanosized phase separations. These aggregations acted as topological constraints and inevitably lead polyester backbone chain closer, namely, the matrix networks stack up more closely. ${ }^{18}$ Therefore, much closer networks of the system can demonstrate higher storage modulus at a room temperature. On the other hand, with the temperature increasing the selfassembled physical aggregations are unjointed gradually. The accumulational network has more free volume which facilitates the network of the system wriggle. As a result, the storage modulus of these resins decreases at a higher rate compared to UPR-S resin as presented in Figure S4.
On the basis of the kinetic theory of rubber elasticity, the experimental cross-link density $\left(n_{\mathrm{e}}\right)$ and the average number of structural units between cross-links $(\nu)$ were determined from the rubbery modulus using the following eq $1^{27}$

$$
G^{\prime}=3 n_{\mathrm{e}} R T, \quad \nu=\frac{\rho}{M_{\mathrm{m}} n_{\mathrm{e}}}
$$

where $G^{\prime}$ represents the storage modulus of the cured resin in the rubbery plateau region, $R$ is the gas constant $\left(8.314 \mathrm{~J} \mathrm{~K}^{-1}\right.$ $\mathrm{mol}^{-1}$ ), $T$ is the absolute temperature in $\mathrm{K}, \rho$ is the density, and $M_{\mathrm{m}}$ is the molecular weight of the structural unit. The kinetic theory of rubber elasticity assumes that the network is ideal and all chains are effective in elastic deformation. ${ }^{27}$ However, because of the complexity of the curing process, a number of network imperfections, like intra-chain loop formation, may occur.

The values of the density at the temperature corresponding to the beginning of the storage modulus rubbery plateau was calculated using the following eq $2^{28}$

$$
\rho(T)=\rho(0) e^{-\beta T}
$$

where $\rho(T)$ and $\rho(0)$ are densities at temperature $T$ and $0{ }^{\circ} \mathrm{C}$, respectively, and $\beta$ is the volumetric temperature expansion coefficient $\left(\mathrm{m}^{3} \mathrm{~m}^{-3}{ }^{\circ} \mathrm{C}^{-1}\right)$. The volumetric temperature expansion coefficient, $\beta$, could be calculated as $\beta=3 \alpha$ assuming the anisotropic nature of UPRs. ${ }^{28}$ The linear temperature expansion coefficient, $\alpha$, was determined by thermomechanical analysis (see below). The calculated values of $n_{\mathrm{e}}$ and $\nu$ are presented in Table 1 . The increase in bulkiness of itaconate side group led to decrease in cross-linking density. This could be explained by cross-linking mechanism which is governed by reactive diluent reactivity. Thus, the cross-linking density of prepared UPRs was the highest for UPR-DMI and the lowest for UPR-DBI. These results were in accordance with experimentally determined gel content. According to the findings obtained from the conducted analysis, we proposed the possible structure of the cured UPRs (Chart 1). The highest reactivity of DMI caused the highest cross-link density 
Table 2. Linear CTE $(\alpha)$, Corresponding Temperature Intervals $(\Delta T)$, and the Average Values of Linear CTE $\left(\alpha_{\text {AV }}\right)$

$\begin{array}{lrccccc}\text { sample } & \Delta T,{ }^{\circ} \mathrm{C} & \alpha, 10-6{ }^{\circ} \mathrm{C}^{-1} & \Delta T,{ }^{\circ} \mathrm{C}^{a} & \alpha, 10-6{ }^{\circ} \mathrm{C}^{-1} & \Delta T,{ }^{\circ} \mathrm{C} & \alpha \mathrm{AV}, 10-6{ }^{\circ} \mathrm{C}^{-1} \\ \text { UPR-S } & 4-48 & 58 & 78-100 & 151 & -20-100 & 96 \\ \text { UPR-DMI } & 7-55 & 54 & 78-100 & 92 & -20-100 & 78 \\ \text { UPR-DEI } & 11-50 & 63 & 81-100 & 139 & -20-100 & 99 \\ \text { UPR-DiPI } & 10-39 & 100 & 64-100 & 169 & -20-100 & 108 \\ \text { UPR-DBI } & 16-95 & 151 & & & -20-100 & 124\end{array}$

${ }^{a} 100{ }^{\circ} \mathrm{C}$ was the last measured temperature so the obtained values of $\alpha$ could be valid for higher temperatures also.

Table 3. Tensile and Compression (Low Stress Mode) Properties of Prepared UPRs

\begin{tabular}{|c|c|c|c|c|c|}
\hline & \multicolumn{3}{|c|}{ tensile test } & \multicolumn{2}{|c|}{ compression } \\
\hline & break stress, $\mathrm{MPa}$ & break stroke Strain, \% & elastic modulus, $\mathrm{MPa}$ & Strain, \% & compression force, $\mathrm{kPa}$ \\
\hline UPR-S & $55.5 \pm 4.3$ & $11.9 \pm 1.1$ & $663 \pm 55$ & 0.219 & 78.0 \\
\hline UPR-DMI & $54.3 \pm 3.8$ & $9.8 \pm 0.9$ & $663 \pm 42$ & 0.206 & 80.0 \\
\hline UPR-DEI & $47.6 \pm 6.2$ & $12.7 \pm 0.8$ & $496 \pm 58$ & 0.314 & 33.8 \\
\hline UPR-DIPI & $33.4 \pm 3.6$ & $15.4 \pm 1.4$ & $390 \pm 32$ & 1.069 & 14.4 \\
\hline UPR-DBI & $20.6 \pm 2.8$ & $12.4 \pm 1.1$ & $268 \pm 33$ & 0.382 & 34.8 \\
\hline
\end{tabular}

of the UPR-DMI (Chart 1b). The cross-linking density decreased with the increase in the length of the ester alkyl group, while the number of dangling side chains increased. Intriguingly, the obtained results for DiPI took notable exception from the established pattern. Namely, despite the significantly lower reported reactivity of DiPI compared to the other investigated dialkyl itaconates, ${ }^{24,25}$ surprisingly high values of $G^{\prime}$, cross-linking density, and $T_{\mathrm{g}}$ for the UPR-DiPI were found. The latter could be explained by the characteristics of the polymerization mechanism of DiPI. Hirano et al. have found that the polymerization of DiPI exhibited the greatest frequency of intramolecular chain-transfer reactions ${ }^{29}$ forming the bulky side chains. These chains additionally strengthened the network by generating internal pressure on polymer chains which increased the overall network tension.

The curves of the dependencies of $\tan \delta$ versus temperature for the UPR networks are presented in Figure S4. The curve maximum, denoting the apparent glass transition temperature, shifted to lower temperatures with an increase in the length of the dialkyl itaconates side group (Table 1). Furthermore, the shape of the $\tan \delta$ curves (broadness and intensity) indicated that the UPR-DMI had the most homogeneous network, whereas the UPR-DiPI had the least homogeneous network. The highest irregularity of the UPR-DiPI network was the consequence of the highest number of dangling chain ends and side chains (Chart 1d) due to the aforementioned specific reactivity of DiPI. The UPR-S had the highest $T_{\mathrm{g}}$ value because of the highest cross-link density and high rigidity of phenyl ring. A shoulder could be noticed on the $\tan \delta$ curves for the UPRDMI, UPR-DEI, and UPR-DiPI. This shoulder might be associated with the $\beta$ relaxation of the thermoset which corresponded to the local molecular motion.

Otsu et al. investigated copolymerization of styrene with various dialkyl itaconates and found that the structure of the alkyl substituents in the itaconate esters does not change the values of the reactivity ratios significantly when styrene is used as a comonomer. ${ }^{25}$ The $r_{\mathrm{S}}$ values were in the range of $0.5-0.7$ while $r$ values of dialyl itaconates were in the range of $0.1-0.2$. Moreover the product of $r_{\mathrm{S}}$ and $r$ values of dialyl itaconates lies in the range of 0.08 to 0.13 , which indicates that the system follows a random distribution. On the basis of the reactivity of the dialkyl itaconates used in this study ${ }^{24}$ similar comonomer distribution could be expected. The assumption of network structure is in accordance with these literature findings.

Bearing in mind that UPRs are mainly used as polymer matrix in various composite materials their coefficient of thermal expansion (CTE) is another important parameter that should be taken into account. If the polymer CTE is too high, an internal stress could be generated at the polymer-metal/ ceramics interface. This stress may further generate distortions and cracks and finally cause the breakage of the material. Therefore, the thermal expansion analysis of these materials is of crucial importance. The linear CTE $(\alpha)$ was calculated from the dependence of strain from temperature presented in Supporting Information (Figure S5) using eq 3

$$
\alpha=\frac{\Delta \varepsilon}{\Delta T}
$$

where $\Delta \varepsilon$ is a strain change in a temperature interval $\Delta T$ in which the change of the strain is linear with temperature. The values of $\alpha$ and the corresponding temperature intervals $(\Delta T)$ are presented in Table 2 . The average values of the linear CTE $\left(\alpha_{\mathrm{AV}}\right)$ for the entire investigated temperature range are also given.

The thermal expansion of UPRs is influenced by numerous factors including the prepolymer molecular weight, the degree of cross-linking, free volume between polymer chains, side groups interaction, and so forth. At lower temperatures, UPR-S and UPR-DMI showed the lowest values of $\alpha$. These lower $\alpha$ values compared to the other samples were attributed to the better chain packing (less free volume) and stronger interactions between phenyl and methyl ester groups. Strong interaction between phenyl groups in UPR-S reduced the chain mobility and therefore reduced thermal expansion. The DMI methyl group in UPR-DMI acted as electron donor and increased the electrostatic interactions of carbonyl group that in turn caused a decrease in the chain mobility of the network. On the other hand, larger side groups sterically hindered the electrostatic interaction of the carbonyl group which increased the chain mobility. Besides this, large side groups prevented good packing of polymer chains increasing the free volume and thus increasing the $\alpha$ corresponding to the UPR-DEI, UPRDiPI, and UPR-DBI.

Temperature increase above the $T_{\mathrm{g}}$ of the corresponding macromolecules consisted of several units of reactive diluents 
(this $T_{\mathrm{g}}$ is much lower than $T_{\mathrm{g}}$ for the corresponding polymer), led to an increase in the $\alpha$. The described change of $\alpha$ above the $T_{\mathrm{g}}$ is common for polymer materials because chain mobility is greatly increased. The highest increase from 58 to $151 \times 10^{-6}$ ${ }^{\circ} \mathrm{C}^{-1}$, was found for the UPR cross-linked with styrene due to the disruption of strong phenyl...phenyl interactions which at lower temperatures strongly prevented chain movement. The UPR-DBI had linear change of $\alpha$ in the temperature range of $0-100{ }^{\circ} \mathrm{C}$ because the $T_{\mathrm{g}}$ of polyDBI of low molecular weight is below $0{ }^{\circ} \mathrm{C} .^{22}$ Also, as it was mentioned before, DBI was found to be the least reactive $\mathrm{RD}$, so the UPR-DBI had the highest number of dangling chain ends (see Chart 1e). Chain ends provide extra free volume because they have more degrees of freedom. ${ }^{30}$ Hence, more chain ends per gram of the sample should imply a higher free volume fraction and therefore higher $\alpha$.

The tensile test results are presented in Table 3. The elastic moduli for all of the synthesized samples were lower compared to the moduli of commercial UPRs that vary from 1 to $3 \mathrm{GPa}^{31}$ The reason for lower values of elastic moduli lies in the fact that prepolymer was synthesized only from aliphatic diacid, itaconic acid. Common acid components used for the synthesis of commercial UPRs are phthalic acid or phthalic anhydride together with maleic anhydride. The exclusion of phthalic acid, and therefore the exclusion of the aromatic component from the prepolymer, led to the decrease in the rigidity of prepolymer chain. As the prepolymer properties, together with cross-link density, have the major influence on the properties of cured UPR, the UPR prepared from aliphatic prepolymer has softer network with lower elastic modulus. Although the elastic moduli of the investigated UPRs were lower compared to commercial ones, this should not be taken as their drawback. Namely, the UPRs with lower elastic moduli and higher elasticity are frequently used in coating industry. Also, UPRs are commonly used as matrix for the synthesis of composite materials wherein the values of the elastic moduli could be greatly increased by the selection of appropriate filler. It is important to notice that the structure of the reactive diluent also affected the tensile properties of the prepared UPRs. The UPR-S sample had the highest value of break stress. The replacement of styrene with itaconates led to decrease in break stress and elastic modulus. Interestingly, the performances of the UPR-DMI sample were very similar to those of UPR-S sample, implying the possibility for the replacement of styrene in the UPR feed composition by biobased monomer. The decrease in tensile properties was more pronounced in the case of itaconates with the increased length of the ester alkyl group due to the lower cross-linking density of these samples (Table 3).

The results obtained from the analysis of the UPRs and cured UPRs (moderate viscosity and elasticity and lower strength) indicated that the novel biobased UPRs could be used for coatings, that is, gel-coats. In such application of UPRs oxygen inhibition commonly occurs during their curing leading to the formation of tacky surface. Thus, it is especially important to investigate this phenomenon if UPRs are intended to be used in coating industry. Although no tacky surfaces were observed after the UPRs curing, the compression tests were carried out under special conditions to investigate the surface mechanical properties. The maximal applied stress was very low $(0.075 \mathrm{MPa})$ causing very small maximal deformations ranging from 5 to $25 \mu \mathrm{m}$, so, only the surface of the samples was affected. The obtained stress versus strain curves are presented in Supporting Information (Figure S6).

The UPR-S and UPR-DMI samples showed practically the same strain versus stress dependence, indicating similar mechanical properties of the surfaces. Bearing in mind that macroscopic mechanical properties (DMA and tensile test) of these two samples were also comparable, the similarity of their surface mechanical properties indicated that oxygen inhibition was similar in both cases. Comparing the macroscopic mechanical properties with the surface mechanical properties of other samples, notable difference could be observed only in the case of the UPR-DiPI. The reason for the lower surface mechanical properties obtained for this sample could be found in the specific reaction mechanism of radical polymerization of DiPI which was more affected by oxygen inhibition.

The thermal degradation of polymeric materials is an important issue from both academic and industrial points of view. TGA measurements provide valuable information that could be used to select materials for certain end-use applications, to predict product performance and useful product lifetimes, and to improve product quality. A comparative thermal gravimetric analysis of synthesized resins is presented in Figure 3. It is shown that the degradation of all resins

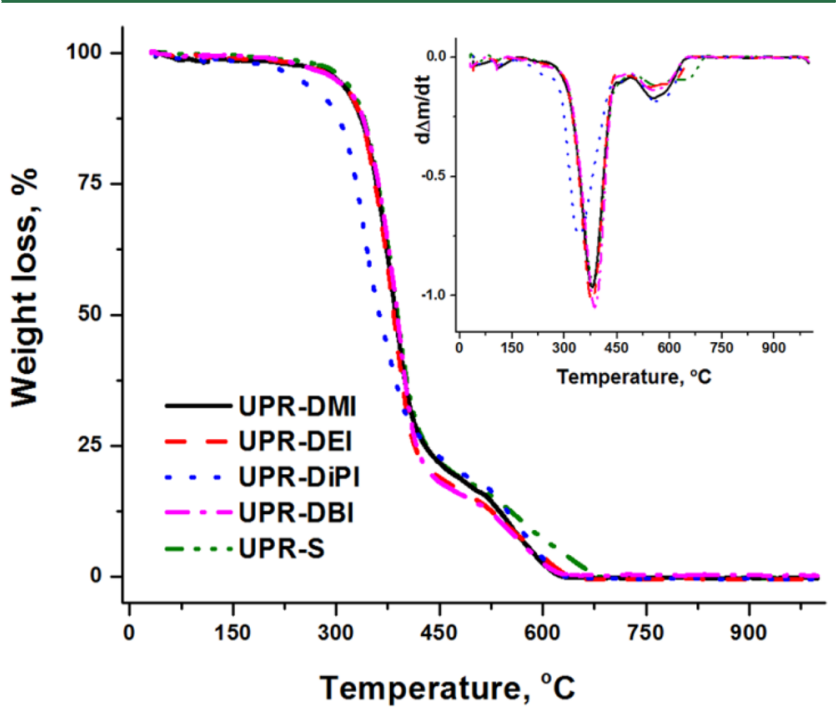

Figure 3. TGA and DTG curves of synthesized resins.

occurred in two steps. The first step was between 265 and 440 ${ }^{\circ} \mathrm{C}$ and may be attributed to the decomposition of the polymer network followed by the release of volatile products and formation of a primary carbonaceous char. ${ }^{32}$ Around $85 \%$ weight loss is observed at the first step. The second step occurred between 485 and $655{ }^{\circ} \mathrm{C}$ and it was associated with the thermal degradation of the carbonaceous char. Also, from Figure 3 it could be observed that there was no significant difference in the thermal stability of synthesized resins except for DiPI, where the first stage was shifted to the lower temperatures. This could be explained by the slightly different decomposition mechanism of polyDiPI compared to the other dialkyl itaconates. ${ }^{33}$ The TGA data implied that UPRs crosslinked with dialkyl itaconates had similar stability and resistance under elevated temperature compared to those cross-linked using styrene.

The majority of studies regarding biobased UPRs deals with the synthesis of prepolymer or $\mathrm{RD}$ from biobased raw materials 


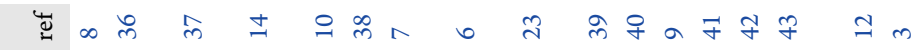

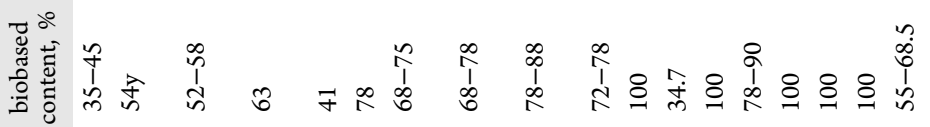

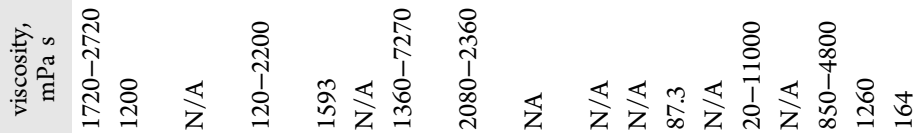

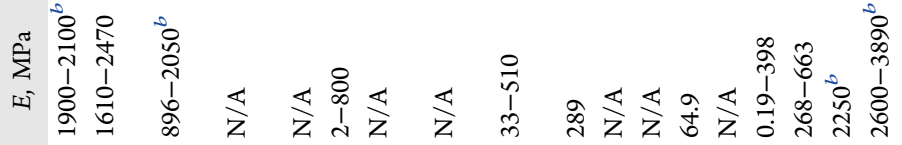

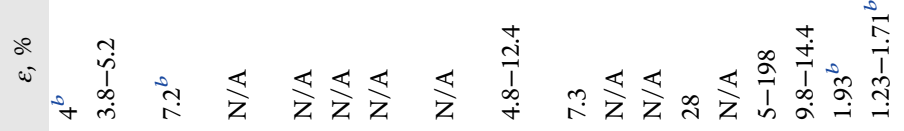

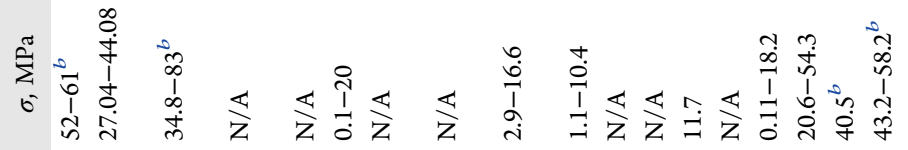

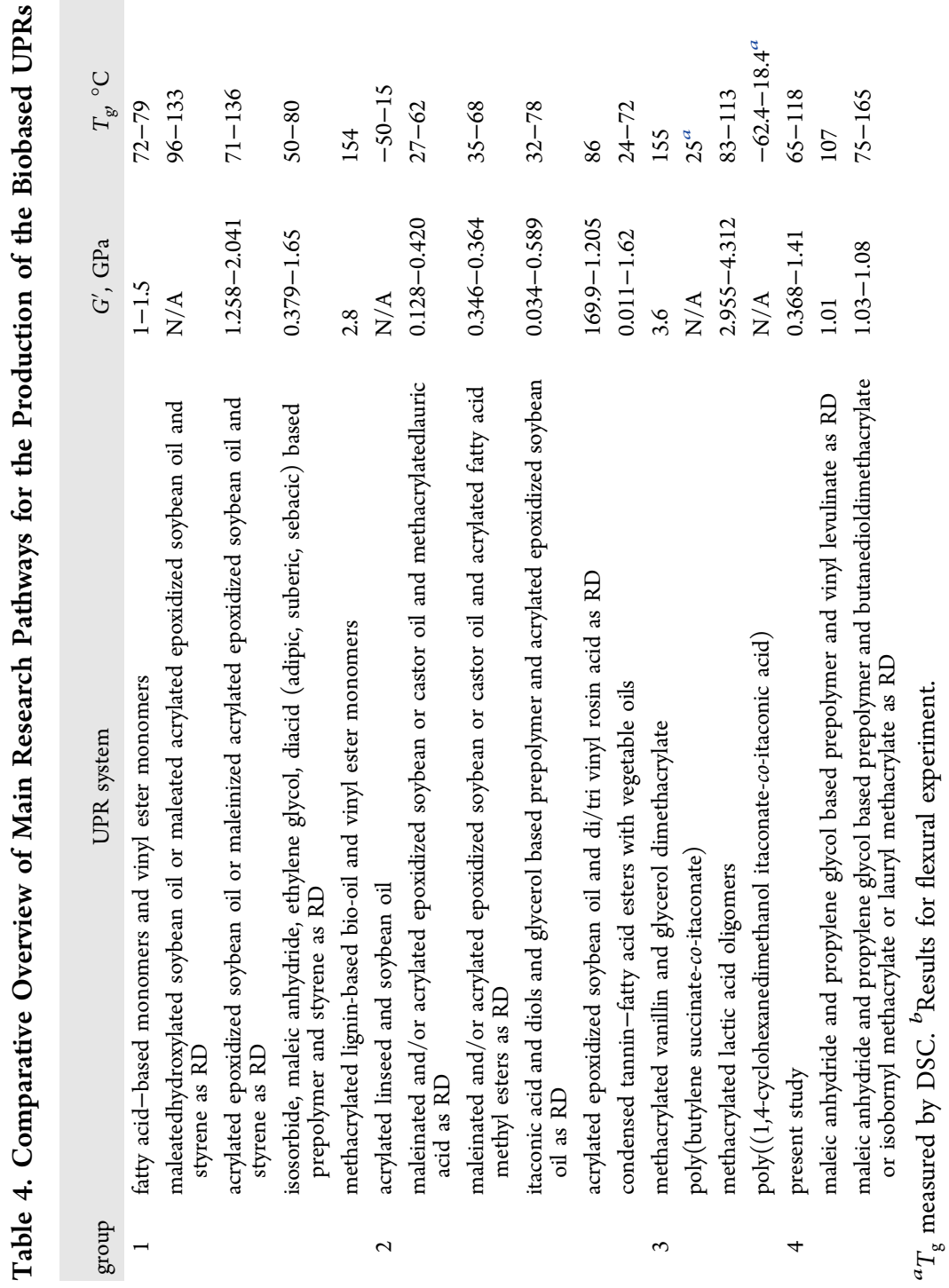


and investigation of several mechanical properties, usually by using tensile and DMA analysis. Thus, it was challenging to perform a detail comparison of our study with previously reported ones. Nevertheless, we gave our best to summarize results of main research pathways and to compare them with our findings. The summarized results are presented in Table 4.

Many studies investigated the usage of acrylated comonomers in the synthesis of UPRs. Although acrylic acid could be fully produced from natural resources, ${ }^{34}$ on the industrial scale this has not been achieved yet. For the studies that have not reported biobased content the presented results were calculated according to the existing data for industrial production. ${ }^{35}$ Also, for the studies that have not investigated tensile properties we included the results of flexural experiments because tensile and flexural properties could be correlated. Generally, in the case of neat UPR flexural strength is around 1.5-3 times higher compared to tensile strength while tensile and flexural modules have similar values.

It is difficult to classify the main approaches to solve the problem of reduction or elimination of petrochemical raw materials in the synthesis of biobased UPRs. This is because similar biobased monomers or oligomers are used in different UPR systems. In order to provide easier and more clarified comparison we separated approaches into four main groups:

1. biobased prepolymer and petrochemical $\mathrm{RD}$

2. prepolymer and/or $\mathrm{RD}$ based on functionalized vegetable oils

3. fully or partly biobased prepolymer, oligomers and/or monomers

4. fully biobased prepolymer and fully or partly biobased RD (modified rosin acid, levulinic acid, itaconic acid, vanillin, etc.)

The studies from group 1 based their investigations on the synthesis of novel biobased prepolymer while using petrochemical RD. As presented in Table 4, UPR from this group showed better applicative properties compared to UPR from other groups indicating that petrochemical $\mathrm{RDs}$ are still superior compared to biobased ones.

Nevertheless, in recent years there has been considerable progress toward the replacement of petrochemical RDs by biobased ones. Many researchers have evaluated vegetable oils or fatty acids as $\mathrm{RD}$ (Group 2). As we mentioned before the main drawbacks of biobased raw materials for the synthesis of UPR are their relatively low reactivity and rather high viscosity. In order to increase reactivity, the vegetable oils and fatty acids have been functionalized, usually acrylated and epoxidized. UPRs from this group possess weaker mechanical properties as a consequence of lower cross-linking density and higher content of aliphatic chains, that is, the lack of stiff aromatic groups. Also, the starting vegetable oil contains fatty acids that are completely saturated and cannot be functionalized with acrylates. So, they act in the same manner as plasticizers, introducing free volume and enabling network to deform more easily. The addition of even small amounts of plasticizer to polymers is known to drastically lower the glass transition temperature and reduce the overall modulus. However, it may be possible to reduce this trend by reducing the amount of the saturated fatty acids. On the other hand, the presence of some saturated fatty acids can contribute to the improved toughness and ballistic impact resistance of UPRs. ${ }^{44}$ Also vegetable oils are the most abundant biobased raw materials and therefore they are cheaper and more available compared to the other biobased RDs.

Other authors have tried to solve problem of higher viscosity by designing a UPR system composed of oligomers and/or monomers (group 3). The fully biobased UPR from this group tends to behave like elastomers having lower ultimate strength, moduli, and $T_{\mathrm{g}}$ and higher elongation at break as a result of lower cross-linking density. The introduction of unsaturation will increase cross-linking density and improve mechanical properties but at the same time lower the biobased content. In this case, considerable care must be paid on the issue of different monomer reactivity. If one monomer is more reactive, it is depleted faster, causing polymer formed later in the reaction to be composed mostly of the slow reacting monomer. This causes heterogeneity in the structure of the polymer network which could greatly reduce mechanical properties.

The studies from group 4 have made an effort to replace styrene by biobased RD while using traditional prepolymer. Many different RDs have been employed with varying success. The main weakness of this approach is peculiar reactivity of fumarate groups with other double bonds which, as mentioned before, could greatly affect mechanical properties. If appropriate $\mathrm{RD}$ is used the mechanical properties of UPR from this group are good but moduli are lower compared to moduli of commercial UPRs. The properties of biobased resins could be significantly improved by the addition of lignin, which introduces required aromatic groups ensuring high stiffness and $T_{\mathrm{g}}$ and further increasing their biobased content with lowcost renewable material. However, issues regarding the lignin solubility and miscibility with various biobased $\mathrm{RDs}$ often occur.

The UPRs synthesized in the present study belong to the group 4. As presented in Table 4, the mechanical properties were generally better compared to other biobased UPRs, but lower compared to UPR where styrene was used as RD. The $T_{\mathrm{g}}$ values were higher compared to other biobased UPRs whereas viscosities were in the same range. From a detailed analysis of data presented in Table 4 one could conclude that the UPRs synthesized in the present study showed equal or better applicative properties compared to other biobased UPRs. However, all of the presented biobased UPRs have lower mechanical properties compared to commercial UPRs. Namely, the commercial resins used in the production of high performance composite materials usually have $G^{\prime}$ in the range of 2-3 GPa, $\sigma$ in the range of 55-75 MPa, $E$ in the range of 2$3 \mathrm{GPa}$, and $T_{\mathrm{g}}$ in the range of $100-150{ }^{\circ} \mathrm{C}$. Although some biobased resins showed high values of moduli and strength, their ductility and resilience were very low limiting their application. ${ }^{3,42}$ Thus, the search for biobased UPRs that could be fully commercially exploited is still on.

\section{CONCLUSION}

On hundred percent biobased UPRs were synthesized by melt polycondensation of itaconic acid with 1,2-propandiol and diluted with dialkyl itaconates. Dimethyl, diethyl, diisopropyl, and dibutyl itaconates, all biobased in nature, with low volatility, excellent miscibility with prepolymer, and compatible viscosity were evaluated as styrene replacement for the synthesis of fully biobased UPRs. An UPR with styrene as $\mathrm{RD}$ was also synthesized and studied as a referent system.

It is shown that all of the dialkyl itaconates copolymerized well with the prepolymer and thus could be effectively used as reactive diluents. DMA analysis showed that cured samples had 
dense and tight network with glass transition temperature in the range of $65-118{ }^{\circ} \mathrm{C}$ and storage moduli in the range of 0.37-1.4 GPa. The cured samples also exhibited moderate stiffness (270-660 MPa) and high break stress (21-54 MPa). It was found that the increase in the length of the itaconate ester group led to the decrease in the mechanical properties as a consequence of lower $\mathrm{RD}$ reactivity and poorer polymer chain packing. The coefficient of the thermal expansion of the resins prepared with dialkyl itaconates was similar to that of the UPR prepared with styrene. The analysis of the overall obtained data indicated that synthesized $100 \%$ biobased resin UPR-DMI had comparable applicative properties to the UPR with styrene, while eliminating toxic and volatile petrochemical components and could be equally used as a matrix for composite materials. Thus, the future work will evaluate the employment of various biobased reinforcements and their effect on UPR-DMI properties.

The reported procedure should encourage the use of biobased resources, such as itaconic acid and its derivatives, in unsaturated polyester resins, as it is a rather straightforward and relatively low-cost approach. The synthesis can be easily conducted using the existing manufacturing equipment and therefore simply up-scaled for industrial production.

\section{ASSOCIATED CONTENT}

\section{S Supporting Information}

The Supporting Information is available free of charge on the ACS Publications website at DOI: 10.1021/acs.biomac.7b00840.

${ }^{13} \mathrm{C}$ NMR spectra of prepolymer (Figure S1), FTIR spectra of prepolymer (Figure S2), GPC chromatogram of prepolymer (Figure S3), The dependences of (a) the storage modulus and (b) the loss tangent of the cured resins on temperature (Figure S4), strain change with temperature for prepared UPRs (Figure S5), stress versus strain curves of prepared UPRs under compressive loading (Figure S6) (PDF)

\section{AUTHOR INFORMATION}

\section{Corresponding Author}

*E-mail: pspasojevic@tmf.bg.ac.rs.

\section{ORCID}

Pavle M. Spasojevic: 0000-0001-8903-2102

\section{Author Contributions}

The manuscript was written through contributions of all authors.

Notes

The authors declare no competing financial interest.

\section{ACKNOWLEDGMENTS}

The authors would like to acknowledge funding from the Ministry of Education, Science, and Technological Development of the Republic of Serbia, through Project No. 172062 "Synthesis and characterization of novel functional polymers and polymeric nanocomposites".

\section{REFERENCES}

(1) NTP (National Toxicology Program). 2016. Report on Carcinogens, Fourteenth Edition.; Research Triangle Park, NC: U.S. Department of Health and Human Services, Public Health Service. http://ntp.niehs.nih.gov/go/roc14/. (Accessed on May 2, 2017).
(2) Report on carcinogens; U.S. Department of Health and Human Services, Public Health Services, 2011; Vol. 12.

(3) Cousinet, S.; Ghadban, A.; Fleury, E.; Lortie, F.; Pascault, J. P.; Portinha, D. Toward replacement of styrene by bio-based methacrylates in unsaturated polyester resins. Eur. Polym. J. 2015, 67, 539-550.

(4) Ma, S.; Li, T.; Liu, X.; Zhu, J. Research progress on bio-based thermosetting resins. Polym. Int. 2016, 65 (2), 164-173.

(5) Vilela, C.; Sousa, A. F.; Fonseca, A. C.; Serra, A. C.; Coelho, J. F. J.; Freire, C. S. R.; Silvestre, A. J. D. The quest for sustainable polyesters - insights into the future. Polym. Chem. 2014, 5 (9), 31193141.

(6) Campanella, A.; La Scala, J. J.; Wool, R. P. The use of acrylated fatty acid methyl esters as styrene replacements in triglyceride-based thermosetting polymers. Polym. Eng. Sci. 2009, 49 (12), 2384-2392.

(7) Campanella, A.; Scala, J. J. L.; Wool, R. P. Fatty acid-based comonomers as styrene replacements in soybean and castor oil-based thermosetting polymers. J. Appl. Polym. Sci. 2011, 119 (2), 10001010.

(8) La Scala, J. J.; Sands, J. M.; Orlicki, J. A.; Robinette, E. J.; Palmese, G. R. Fatty acid-based monomers as styrene replacements for liquid molding resins. Polymer 2004, 45 (22), 7729-7737.

(9) Stanzione Iii, J. F.; Sadler, J. M.; La Scala, J. J.; Reno, K. H.; Wool, R. P. Vanillin-based resin for use in composite applications. Green Chem. 2012, 14 (8), 2346-2352.

(10) Stanzione, J. F.; Giangiulio, P. A.; Sadler, J. M.; La Scala, J. J.; Wool, R. P. Lignin-Based Bio-Oil Mimic as Biobased Resin for Composite Applications. ACS Sustainable Chem. Eng. 2013, 1 (4), 419-426.

(11) Stanzione, J. F.; Sadler, J. M.; La Scala, J. J.; Wool, R. P. Lignin Model Compounds as Bio-Based Reactive Diluents for Liquid Molding Resins. ChemSusChem 2012, 5 (7), 1291-1297.

(12) Cousinet, S.; Ghadban, A.; Allaoua, I.; Lortie, F.; Portinha, D.; Drockenmuller, E.; Pascault, J.-P. Biobased vinyl levulinate as styrene replacement for unsaturated polyester resins. J. Polym. Sci. J. Polym. Sci., Part A: Polym. Chem. 2014, 52 (23), 3356-3364.

(13) Besse, V.; Camara, F.; Voirin, C.; Auvergne, R.; Caillol, S.; Boutevin, B. Synthesis and applications of unsaturated cyclocarbonates. Polym. Chem. 2013, 4 (17), 4545-4561.

(14) Sadler, J. M.; Toulan, F. R.; Nguyen, A.-P. T.; Kayea, R. V.; Ziaee, S.; Palmese, G. R.; La Scala, J. J. Isosorbide as the structural component of bio-based unsaturated polyesters for use as thermosetting resins. Carbohydr. Polym. 2014, 100, 97-106.

(15) Lewis, F. M.; Walling, C.; Cummings, W.; Briggs, E. R.; Mayo, F. R. Copolymerization. IV. Effects of Temperature and Solvents on Monomer Reactivity Ratios. J. Am. Chem. Soc. 1948, 70 (4), 15191523.

(16) Lewis, F. M.; Mayo, F. R. Copolymerization. IX. A Comparison of Some cis and trans Isomers1,2. J. Am. Chem. Soc. 1948, 70 (4), $1533-1536$

(17) Matsumoto, A.; Kamigaki, H.; Oiwa, M. Studies of the polymerization of diallyl compounds. XXXVII. Copolymerizations of diallyl phthalate with dialkyl fumarates. J. Polym. Sci., Polym. Chem. Ed. 1982, 20 (9), 2611-2620.

(18) Farmer, T. J.; Clark, J. H.; Macquarrie, D. J.; Ogunjobi, J. K.; Castle, R. L. Post-polymerisation modification of bio-derived unsaturated polyester resins via Michael additions of 1,3-dicarbonyls. Polym. Chem. 2016, 7 (8), 1650-1658.

(19) Lv, A.; Li, Z.-L.; Du, F.-S.; Li, Z.-C. Synthesis, Functionalization, and Controlled Degradation of High Molecular Weight Polyester from Itaconic Acid via ADMET Polymerization. Macromolecules 2014, 47 (22), 7707-7716.

(20) Farmer, T.; Castle, R.; Clark, J.; Macquarrie, D. Synthesis of Unsaturated Polyester Resins from Various Bio-Derived Platform Molecules. Int. J. Mol. Sci. 2015, 16 (7), 14912.

(21) Schute, K.; Detoni, C.; Kann, A.; Jung, O.; Palkovits, R.; Rose, M. Separation in Biorefineries by Liquid Phase Adsorption: Itaconic Acid as Case Study. ACS Sustainable Chem. Eng. 2016, 4 (11), 59215928. 
(22) Sarkar, P.; Bhowmick, A. K. Green Approach toward Sustainable Polymer: Synthesis and Characterization of Poly(myrcene-co-dibutyl itaconate). ACS Sustainable Chem. Eng. 2016, 4 (4), 2129-2141.

(23) Dai, J.; Ma, S.; Wu, Y.; Han, L.; Zhang, L.; Zhu, J.; Liu, X. Polyesters derived from itaconic acid for the properties and bio-based content enhancement of soybean oil-based thermosets. Green Chem. 2015, 17 (4), 2383-2392.

(24) Sato, T.; Hirose, Y.; Seno, M.; Tanaka, H.; Uchiumi, N.; Matsumoto, M. Kinetic and ESR studies on radical polymerization. Radical polymerization of diisopropyl itaconate. Eur. Polym. J. 1994, 30 (3), 347-352.

(25) Sato, T.; Takahashi, Y.; Seno, M.; Nakamura, H.; Tanaka, H.; Ota, T. Effect of alkyl groups on the rate constants of propagation and termination in the radical polymerization of dialkyl itaconates. Makromol. Chem. 1991, 192 (12), 2909-2914.

(26) Cowie, J. M. G.; Arrighi, V. Polymers: chemistry and physics of modern materials; CRC Press, 2007.

(27) Mark, J. E. Experimental Determinations of Crosslink Densities. Rubber Chem. Technol. 1982, 55 (3), 762-768.

(28) Orwoll, R. Densities, Coefficients of Thermal Expansion, and Compressibilities of Amorphous Polymers. In Physical Properties of Polymers Handbook; Mark, J., Ed.; Springer: New York, 2007; pp 93101.

(29) Hirano, T.; Furuya, K.; Seno, M.; Sato, T. 13C NMR and electron spin resonance analyses of the radical polymerization of diisopropyl itaconate. J. Polym. Sci., Part A: Polym. Chem. 2002, 40 (24), 4513-4522.

(30) Fox, T. G.; Flory, P. J. The glass temperature and related properties of polystyrene. Influence of molecular weight. J. Polym. Sci. 1954, 14 (75), 315-319.

(31) Kandelbauer, A.; Tondi, G.; Zaske, O. C.; Goodman, S. H. Unsaturated Polyesters and Vinyl Esters. In Handbook of Thermoset Plastics, third ed.; William Andrew Publishing: Boston, 2014; pp 111172.

(32) Tibiletti, L.; Longuet, C.; Ferry, L.; Coutelen, P.; Mas, A.; Robin, J.-J.; Lopez-Cuesta, J.-M. Thermal degradation and fire behaviour of unsaturated polyesters filled with metallic oxides. Polym. Degrad. Stab. 2011, 96 (1), 67-75.

(33) Bošković, G.; Katsikas, L.; Veličković, J. S.; Popović, I. G. The thermal degradation of poly(di-n-propyl itaconate) and poly(di-isopropyl itaconate). Polymer 2000, 41 (15), 5769-5775.

(34) Chu, H. S.; Ahn, J.-H.; Yun, J.; Choi, I. S.; Nam, T.-W.; Cho, K. M. Direct fermentation route for the production of acrylic acid. Metab. Eng. 2015, 32, 23-29.

(35) Bio-based products. Bio-based carbon content. Determination of the bio-based carbon content using the radiocarbon method. In 2014; Vol. TC 411.

(36) Lu, J.; Wool, R. P. Sheet molding compound resins from soybean oil: Thickening behavior and mechanical properties. Polym. Eng. Sci. 2007, 47 (9), 1469-1479.

(37) Lu, J.; Hong, C. K.; Wool, R. P. Bio-based nanocomposites from functionalized plant oils and layered silicate. J. Polym. Sci., Part B: Polym. Phys. 2004, 42 (8), 1441-1450.

(38) La Scala, J.; Wool, R. P. Property analysis of triglyceride-based thermosets. Polymer 2005, 46 (1), 61-69.

(39) Ma, Q.; Liu, X.; Zhang, R.; Zhu, J.; Jiang, Y. Synthesis and properties of full bio-based thermosetting resins from rosin acid and soybean oil: the role of rosin acid derivatives. Green Chem. 2013, 15 (5), 1300-1310.

(40) Luo, C.; Grigsby, W. J.; Edmonds, N. R; Al-Hakkak, J. Vegetable oil thermosets reinforced by tannin-lipid formulations. Acta Biomater. 2013, 9 (2), 5226-5233.

(41) Jiang, Y.; van Ekenstein, G. O. R. A.; Woortman, A. J. J.; Loos, K. Fully Biobased Unsaturated Aliphatic Polyesters from Renewable Resources: Enzymatic Synthesis, Characterization, and Properties. Macromol. Chem. Phys. 2014, 215 (22), 2185-2197.

(42) Bakare, F. O.; Åkesson, D.; Skrifvars, M.; Bashir, T.; Ingman, P.; Srivastava, R. Synthesis and characterization of unsaturated lactic acid based thermoset bio-resins. Eur. Polym. J. 2015, 67, 570-582.
(43) Barrett, D. G.; Merkel, T. J.; Luft, J. C.; Yousaf, M. N. One-Step Syntheses of Photocurable Polyesters Based on a Renewable Resource. Macromolecules 2010, 43 (23), 9660-9667.

(44) Wool, R. P. Polymers and Composite Resins from Plant Oils. In Bio-Based Polymers and Composites; Wool, R. P., Ed.; Elsevier Academic Press: London, 2005; pp 56-113. 